\title{
Collecting State-level Oral Health Data When Resources Are Limited: an Approach to Oral Health Surveillance
}

\author{
Susan G. Reed, DDS, MPH \\ School of Public Health \\ Department of Community Health Programs \\ Program in Dental Public Health \\ University of Michigan at Ann Arbor \\ Ann Arbor, Ml 48109-2029 \\ Jacqueline A. Tallman, RDH, MPA \\ Oral Health Program Coordinator \\ Michigan Department of Public Health \\ Lansing, MI \\ Brian A. Burt, BDSc, MPH, PhD \\ Chairman and Professor \\ School of Public Health \\ Department of Community Health Programs \\ Program in Dental Public Health \\ University of Michigan at Ann Arbor \\ Ann Arbor, MI
}

adults examined, 45.1 percent of 20-64-year-olds $(n=820)$ and 38.1 percent of those 65 years or older $(n=105)$ had two or more teeth with untreated decay. These data suggest a high level of untreated disease among the service populations of the local dental health agencies. The method of data collection was well accepted by the staff of the agencies concerned and proved to be simple and inexpensive to conduct. While the population examined is not representative of the state population, it reflects a good cross-section of the population seeking clinical dental services through public health agencies. The data will be used in program development and to address the Maternal and Child Health reporting requirements of the Omnibus Budget Reconciliation Act of 1989. We intend to conduct this oral health surveillance survey periodically in Michigan to demonstrate trends in oral disease.

Key Words: dental caries, dental clinics, oral health surveys, local dental public health, public health dentistry.

The periodic collection of oral disease data is a fundamental aspect of dental public health practice at the state and local levels. These data are considered an integral part of the planning, monitoring, and evaluation functions of a state-level agency as described by Siegal et al. (1). More recently, the need for data on oral conditions has been given greater urgency by the federal requests 
for statewide dental needs assessments to complete Maternal and Child Health (MCH) Block Grant applications and to address the data requirements of the Omnibus Budget Reconciliation Act (OBRA) of 1989. But like its counterparts in many other states, the Oral Health Program of the Michigan Department of Public Health (MDPH) lacks sufficient resources to conduct statewide surveys of the "classical" variety, meaning those with clinical examinations of representative samples carried out by trained examiners according to a standard protocol. So instead, Michigan has been seeking an efficient method of collecting oral health data that will meet the $\mathrm{MCH}$ requirements, and that will also provide data of acceptable quality for planning and evaluation. We initiated this oral health surveillance system as a response to this need. It is a low-cost method for local dental agencies to use in profiling the oral status of the service population, and is simple enough in both concept and execution to be repeated at periodic intervals.

This paper describes the development of the oral health surveillance system and the results of its first application in Michigan during 1991. The method collected information only on dental caries during this first application, although it can be extended to other oral conditions in time. The primary purpose of this first survey was to determine the extent of untreated dental caries among the service populations of the local agencies.

\section{Methods}

Every publicly supported local agency in Michigan providing clinical dental services was considered eligible, except those dental clinics affiliated with the schools of dentistry and dental hygiene, general practice residencies, and a few small local programs operating solely on private resources. There were 20 agencies meeting the inclusion criteria. These agencies, which served 28 of Michigan's 83 counties, included 16 county health department dental clinics, two Indian Health Service (IHS) dental clinics, one dental hygiene program serving home-bound patients, and one hospital-based dental clinic. Each agency was asked to record data on dental caries from all patients of all ages who presented for an initial or recall examination over a specified four-week period during the spring of 1991. Emergency patients and those already receiving treatment were excluded. Thus, the design was a descriptive cross-sectional study using nonprobability consecutive patient sampling for the designated weeks.

Data were collected only for decayed or filled primary and permanent teeth. Because teeth can be missing for reasons not related to caries, and because of the focus on untreated caries, missing teeth were excluded from this study. For patients aged six years and older, only the permanent dentition was recorded. Additional data collected were: nursing caries in 2-5-year-olds, sealants and surface-specific caries status for first permanent molars in 6-19-year-olds, and root caries and presence of two or more teeth with untreated decay in adults. Instructions on use of the data record and a protocol for diagnostic criteria were sent to the agencies prior to the examination period. The diagnostic criteria for coronal and root caries were those used by the National Institute for Dental Research in its national surveys $(2,3)$. The definition for nursing caries (baby bottle tooth decay) was that used by Kelly and Bruerd (4)-as the presence of at least three primary maxillary incisors affected by dental caries modified for this survey to include unaffected mandibular incisors.

The protocol required clinical and questionnaire data to be collected by either a dentist or hygienist in the local agency at the time the patient was examined. The examiner recorded the following data for each patient seen: (1) appointment type, (initial or recall); (2) number of teeth present ever affected by dental caries (i.e., either decayed or filled); (3) number of teeth with untreated decay; and (4) a yes/no response for the presence of nursing caries (for 2-5-year-olds only), the presence of at least one sealant (6-19-year-olds only), and the presence of at least one tooth with root caries (20-64- and 65+year-olds). On the record form for the 6-19-year-olds, four supplemental spaces were available to supply surface-specific data on the first permanent molars. Copies of the record forms are available from the authors.

There were four separate record forms, one for each of four specified age ranges. Each form had space to record information for 20 patients. While target age groups for data presentation can be chosen as needed, those selected for this survey were 2-5-year-olds, 6-19-year-olds, 20-64year-olds, and $65+$ year-olds. The procedures were pilottested at three agencies, and a number of modifications made to the forms and the data-collection process as a result. The dental agencies were requested to mail their completed forms to the Michigan Department of Public Health, Oral Health Program, for analysis.

Summary totals from the record forms were entered into a spreadsheet program by agency and by age groups for descriptive analysis. Data were pooled for statewide analysis, and the local agencies later received a summary of their own data with the pooled results as a comparison.

\section{Results}

Completed records were returned by 19 of the 20 agencies contacted. The data are mostly from the month of June, with one agency collecting in September 1991. Over the four-week observation period, data were collected for a total of 1,976 patients from the 19 agencies. A postsurvey questionnaire showed that the relative number of patients examined and the amount of dental disease recorded during the examination period approximated an average monthly figure for the pooled data. Table 1 shows the distribution of patients by age group 
TABLE 1

Distribution of Patients by Age Group and Examination Type

\begin{tabular}{lccc}
\hline $\begin{array}{l}\text { Age Range } \\
\text { (Years) }\end{array}$ & $n$ & $\begin{array}{l}\text { Initial } \\
\text { Exam }\end{array}$ & $\begin{array}{c}\text { Recall } \\
\text { Exam }\end{array}$ \\
\cline { 2 - 4 } $2-5$ & 341 & 246 & 95 \\
$6-19$ & 710 & 212 & 498 \\
$20-64$ & 820 & 255 & 565 \\
$65+$ & 105 & 10 & 95
\end{tabular}

and by initial and recall examination.

Among the 2-5-year-olds 40.5 percent had some caries experience, 35.2 percent overall with untreated decay. Equivalent figures for permanent teeth in the 6-19-yearolds were 61.4 percent and 40.4 percent. Distribution of untreated decayed primary teeth in preschool children is shown in Figure 1, and the distribution of untreated decayed permanent teeth for 6-19-year-olds in Figure 2. The skewed distributions demonstrate that while many children have few or no untreated lesions, significant numbers in both groups have a substantial number of untreated carious lesions. Table 2 presents summary information for patients with caries experience by age group, and illustrates the relatively high severity among those affected by caries. Among the 35.2 percent of preschoolers with untreated decay, the mean number of decayed teeth was $4.3(\mathrm{SD} \pm 3.5)$, and the $\mathrm{d} / \mathrm{dft}$ ratio was 78.2 percent. Nursing caries was noted at chairside for 10 percent $(34 / 341)$ of the $2-5$-year-olds. For the 40.4 percent of 6-19-year-olds with untreated decay, the mean number of decayed permanent teeth was $2.7(\mathrm{SD} \pm 2.1)$, and the D/DFT ratio was 41.8 percent. At least one sealant was present in $116 / 710(16.3 \%)$ of the $6-19$-year-olds. Of the 2,482 first permanent molars examined, $1,012(40.8 \%)$ had caries experience. Of these molars with caries experience, 86.5 percent had only occlusal, buccal, lingual involvement, while the remainder had proximal surfaces affected.

In the 20-64-year-old age group, 59.1 percent of the patients had untreated caries and 45.1 percent had two or more teeth with decay. The mean was $4.4(\mathrm{SD} \pm 3.9)$ carious teeth for those with any decayed teeth. The proportion of patients with at least one decayed or filled root surface was 10.7 percent. For the group of patients aged 65 or more years, 62.9 percent had untreated caries, 38.1 percent had two or more teeth with decay, and 57.1 percent had at least one decayed or filled root surface.

\section{Discussion}

While there has always been lip service to the principle of basing program planning on survey data, state- or local-level planning frequently has gone ahead with data that are obsolete, generalized from national or regional surveys, or even without the use of data at all. This sort
FIGURE 1

Distribution of Untreated Carious Primary Teeth in 2-5-year-olds $(n=341)$

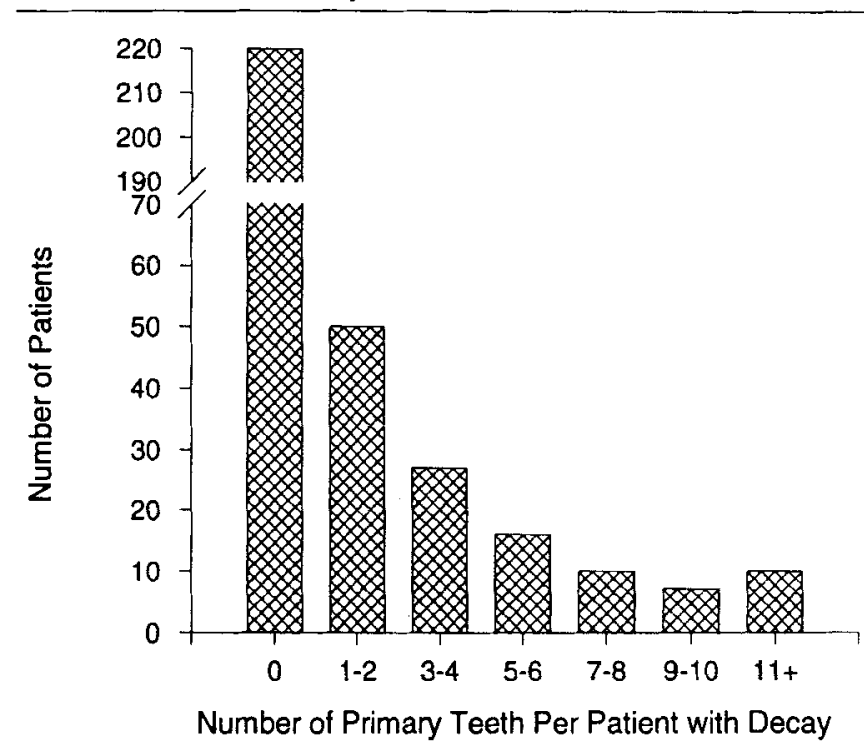

FIGURE 2

Distribution of Untreated Carious Permanent Teeth in 6-19-year-olds $(n=710)$

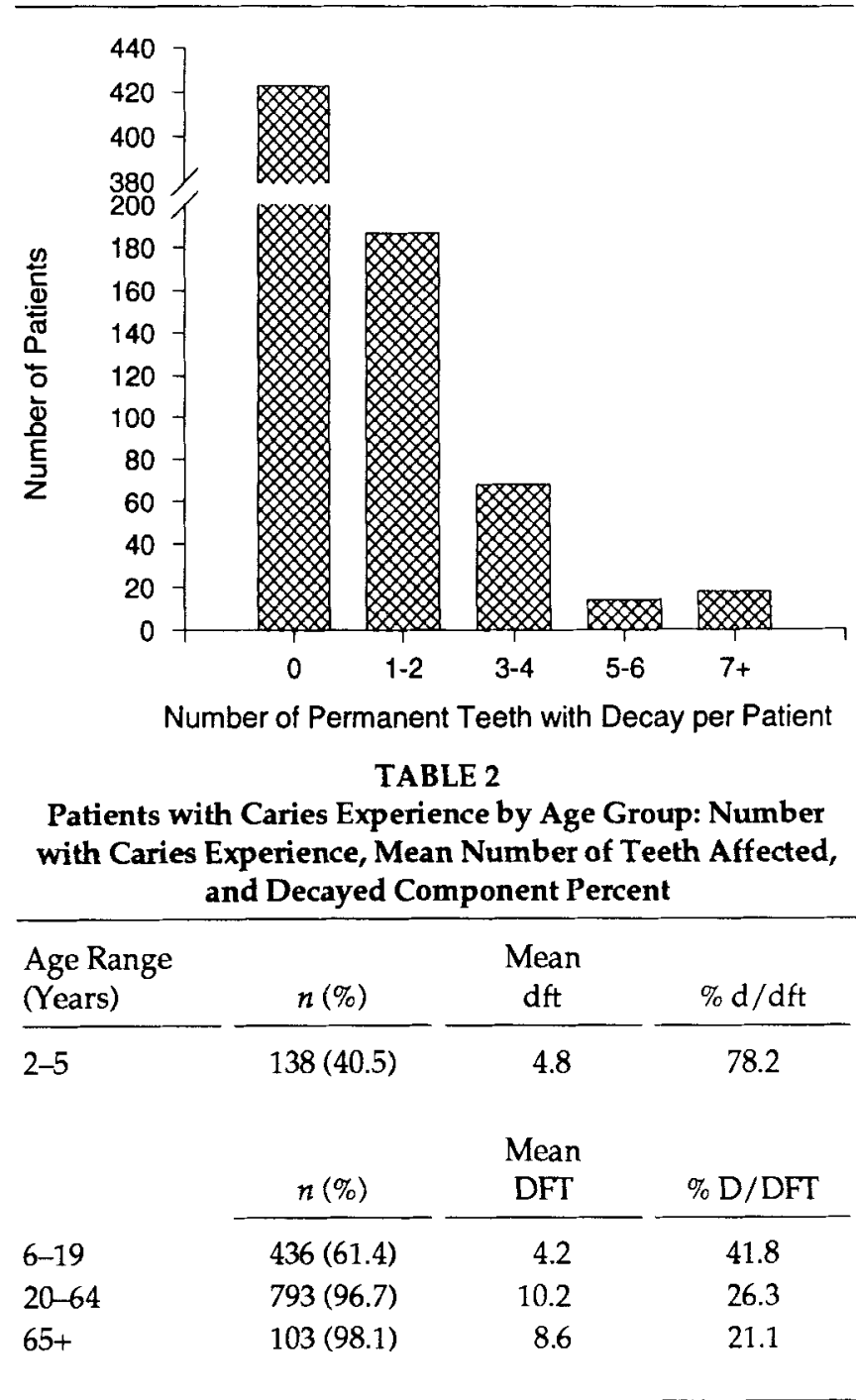


of planning was more or less justified on the grounds that there were no resources to carry out surveys, the problems demanding attention were obvious, or a survey would take too long to conduct and analyze. OBRA, however, has injected a sense of real-world urgency into the need for data collection and analysis. OBRA requires states receiving funds from the MCH Block Grant to justify that these funds are well spent by providing evaluatory data from the programs that use them. Since many state dental programs (in addition to other health programs) are funded from $\mathrm{MCH}$ Block Grants, they are included in these requirements. As a result, survey data have now become a necessity for many. For those dental programs for whom conducting a "classical" survey (i.e., representative samples, trained examiners, a generous budget) is not an option, there is a problem of how the needed data are to be collected.

The full range of information available from the data recorded in this pilot is displayed in Figure 3. This approach is essentially pragmatic: some data, even if limited in their application, are better than no data. This philosophy has been embodied for some years in the Pathfinder survey protocol (5) of the World Health Organization ( $\mathrm{WHO}$ ), an approach intended to collect data for administrative purposes, but that deviates from the design rigor associated with textbook surveys. In particular, WHO's purposive sampling procedures for a Pathfinder survey contrast sharply with morecomplicated representative sampling techniques. The Pathfinder protocol has been developed with third-world conditions in mind, and there is little doubt that it has stimulated the collection of a great deal of oral health data-now stored in WHO's Global Oral Data Bank-that would not have been collected without it (6). A more sophisticated application of the same philosophy is seen in the periodic reports from the British Association for the Study of Community Dentistry $(7,8)$. In Britain, dentists in the community services use a standard protocol to collect data that allow geographic comparisons and are used to assess trends over time.

The limitations of applying data collected under this approach to program planning and evaluation must be recognized clearly. Some authorities, in fact, subscribe to a countervailing philosophy, which states that use of limited data can be dangerously misleading, that unless data from "classical" surveys can be used, then it is better to use no data. We do not subscribe to this view. We recognize that problems can arise when Pathfinder data are given the same weight as more rigorously controlled data in analytical epidemiology, but such "stretching" of the data beyond their limitations is a misuse rather than an inherent flaw in the Pathfinder approach itself. Use of a quick and inexpensive form of data collection, while usually insufficient for analytic epidemiology, can provide information that is sufficiently valid to identify problems and priorities for planning and evaluating pub-
FIGURE 3

Summary Data Retrievable from Oral Health Surveillance Records

Date (month/year) of examination

Number of patients examined, percent initial and percent recall

\section{Caries Summary-primary or permanent teeth}

Number of teeth with caries experience (affected by caries, presently treated or untreated)

Number of teeth with decay, percent decay of caries experience

Number of patients with caries experience, percent caries-free Average caries experience of patients with caries experience Average decay experience of patients with caries experience

\section{Age group specific information}

2-5-year-olds

Number of patients with nursing caries

6-19-year-olds

Number of patients with at least one sealant present

Number of first permanent molars, percent with caries experience

Number, percent with only occlusal, buccal, lingual surfaces affected

Number, percent with proximal surfaces affected $20-64-65+$ year-olds

Number of patients with at least one decayed or filled root surface

Number of patients with two or more teeth with decay

lic health programs administered at this service level. When limited data are all that are available, they should be used in program administration to the extent possible, though with a clear recognition of their limitations. These limitations include a sampling design restricted to the service population of local health agencies, so results cannot be generalized, nor sampling error be determined. In addition, examiners are not trained beforehand, and there is little control over the quality of data collected. On the other hand, the chance of bias influencing sample selection has been excluded as far as possible, so it is likely that those examined represent the service population fairly closely.

While direct comparisons of our results with national survey data are not justified, the similar shape of the distributions of untreated carious teeth (Figures 1 and 2) to those with caries experience in the national survey data (9) strengthen the likelihood that the sample seen in this survey is a fair representation of the target population. Oral health surveillance data, like the British data referred to earlier, could also be used to compare geographic conditions from one agency to another when the numbers are high enough (they were not in this pilot). Perhaps the most valuable aspect of this approach, however, is that it will permit trends to be discerned when its 
protocol is followed in subsequent surveys.

The oral heal th surveillance system has been designed for ease of analysis. The system allows for individual patient data entry rather than only using the summary totals from the form. Using the forms in this manner provides more analytical options-frequency distributions of untreated decay and standard deviations can be calculated. In the future, we expect that computerized direct data entry will enable more sophisticated analysis and eliminate the need for the paper record form, thus reducing the overall analytical task even further.

Data were to be recorded with the patient present; from inquiries made to each of the agencies, this requirement presented no difficulties. An alternative method employed by a few agencies was to record the data at a later time from the patients' dental charts. Most who collected the data and completed the record forms reported that the amount of time and effort necessary to produce the information was not a problem. One notable fringe benefit of the survey was the positive involvement of local health department personnel. They demonstrated an unexpectedly high degree of enthusiasm for taking part in the survey, probably because they could see its utility and the likely benefits to their own local programs. We intend to continue the oral health surveillance survey in Michigan on an annual or biennial basis.

\section{Acknowledgments}

The authors thank the staff and dental care practitioners at the local health agencies of Michigan for their cooperation and assistance. The data presented do not singly reflect any one agency or the Indian Health Service.

\section{References}

1. Siegal MD, Martin B, Kuthy RA. Usefulness of a local oral health survey in program development. J Public Health Dent 1988;48:1214.

2. US Public Health Service, National Institutes of Dental Research. Oral health of United States children. Washington, DC: Government Printing Office, 1989; NIH pub no 89-2247.

3. US Public Health Service, National Institutes of Dental Research. Oral health of United States adults: regional findings. Washington, DC: Government Printing Office, 1988; NIH pub no 88-2869.

4. Kelly M, Bruerd B. The prevalence of baby bottle tooth decay among two Native American populations. J Public Health Dent 1987;47(2): $94-7$.

5. World Health Organization. Oral health surveys: basic methods. 3rd ed. Geneva: WHO, 1987.

6. Pilot T, Barmes DE, Leclerc MH, McCombie BJ, Sardo Infirri J. Periodontal conditions in adolescents, 15-19 years of age: an overview of CPITN data in the WHO Global Oral Data Bank. Community Dent Oral Epidemiol 1987;15:336-8.

7. Dowell TB, Evans DJ. The dental caries experience of 5-year-old children in Great Britain. A survey coordinated by the British Association for the Study of Community Dentistry in 1987-88. Community Dent Health 1989;6:271-9.

8. Evans DJ, Dowell TB. The dental caries experience of 12-year-old children in Great Britain. A survey coordinated by the British Association for the Study of Community Dentistry in 1988-89. Community Dent Health 1990;7:307-14.

9. Burt BA, EklundSA. Dentistry, dental practice, and the community. 4th ed. Philadelphia: Saunders, 1992:97. 